\title{
3D Pick \& Mix: Object Part Blending in Joint Shape and Image Manifolds
}

\author{
Adrian Penate-Sanchez ${ }^{1,2}[0000-0003-2876-3301]$ \\ Lourdes Agapito ${ }^{10000-0002-6947-1092]}$ \\ 1 University College London \\ Department of Computer Science, United Kingdom \\ ${ }^{2}$ University of Oxford \\ Oxford Robotics Institute, United Kingdom \\ adrian@robots.ox.ac.uk ${ }^{\star}$
}

\begin{abstract}
We present 3D Pick \& Mix, a new 3D shape retrieval system that provides users with a new level of freedom to explore $3 \mathrm{D}$ shape and Internet image collections by introducing the ability to reason about objects at the level of their constituent parts. While classic retrieval systems can only formulate simple searches such as "find the $3 D$ model that is most similar to the input image" our new approach can formulate advanced and semantically meaningful search queries such as: "find me the $3 D$ model that best combines the design of the legs of the chair in image 1 but with no armrests, like the chair in image 2". Many applications could benefit from such rich queries, users could browse through catalogues of furniture and pick and mix parts, combining for example the legs of a chair from one shop and the armrests from another shop.
\end{abstract}

Keywords: Shape blending · Image embedding · Shape retrieval

\section{Introduction}

As databases of images and 3D shapes keep growing in size and number, organizing and exploring them has become increasingly complex. While most tools so far have dealt with shape and appearance modalities separately, some recent methods [15]10, have begun to exploit the complementary nature of these two sources of information and to reap the benefits of creating a common representation for images and 3D models. Once images and 3D shapes are linked together, many possibilities open up to transfer what is learnt from one modality to another. Creating a joint embedding allows to retrieve 3D models based on image queries (or vice-versa) or to align images of similar 3D shapes. However, recent retrieval methods still fall short of being flexible enough to allow advanced queries. Crucially, they are limited to reasoning about objects as a whole - taking a single query image (or shape) as input at test time prevents them from

\footnotetext{
* This work was supported by the SecondHands project, funded from the EU Horizon 2020 Research and Innovation programme under grant agreement 643950 and by the EPSRC grants RAIN and ORCA (EP/R026084/1, EP/R026173/1).
} 


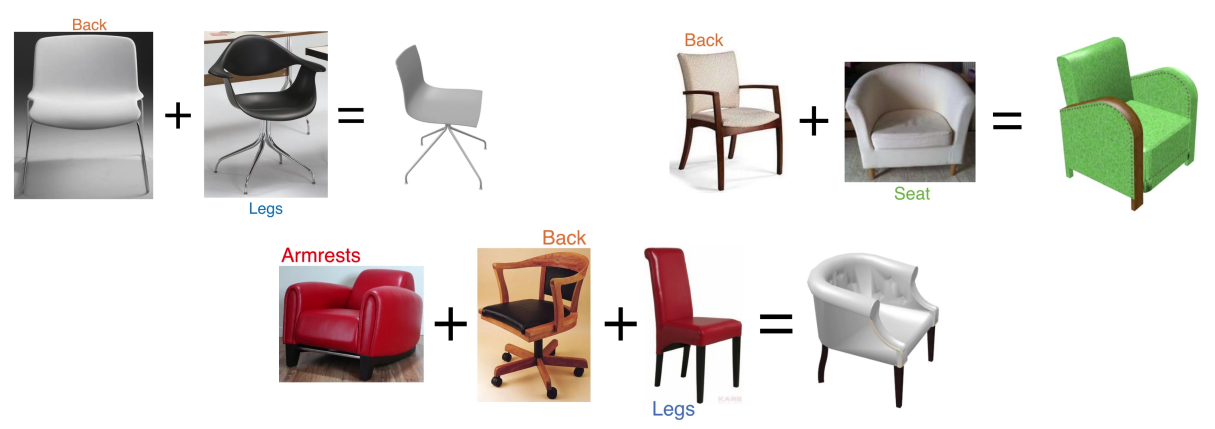

Fig. 1. Our approach takes two (or more) inputs, either RGB images or 3D models, and part labels such as "legs" or "backrest" and retrieves a shape that combines object parts or properties from all inputs, via a cross-manifold optimization technique.

combining object properties from different inputs. 3D Pick \& Mix overcomes this limitation by reasoning about objects at the level of parts. It can formulate advanced queries such as: "find me the 3D model that best combines the design of the backrest of the chair in image 1 with the shape of the legs of the chair in image 2" (see Fig. 1) or "retrieve chairs with wheels". The ability to reason at the level of parts provides users with a new level of freedom to explore 3D shape and image datasets. Users could browse through catalogues of furniture and pick and mix parts, combining for example the legs of a favourite chair from one catalogue and the armrests from another (see Fig. 11).

Our system first builds independent manifold shape spaces for each object part (see Fig. 4). A CNN-based manifold coordinate regressor is trained to map real images of an object to the part manifolds. Our novel deep architecture jointly performs semantic segmentation of object parts and coordinate regression for each part on the corresponding shape manifold. This network is trained using only synthetic data, Fig. 2 illustrates the architecture. At test time the user provides two (or more) images (or 3D models) as input and determines which parts they would like to pick from each (note that this only requires a label name such as 'legs'). Our system retrieves the model that best fits the arrangement of parts by performing a cross-manifold optimization (see Fig. 1). The main contributions of our 3D Pick \& Mix system are:

- We learn embeddings (manifolds) for object parts (for instance the legs or the armrests of chairs) which allow us to retrieve images or 3D models of objects with similarly shaped parts.

- We propose a new deep architecture that can map RGB images onto these manifolds of parts by regressing their coordinates. Crucially, the input to the network is simply an RGB image and the name (label) of the object part. The CNN learns to: (i) segment the pixels that correspond to the chosen part, and (ii) regress its coordinates on the shape manifold.

- At query time our retrieval system can combine object parts from multiple input images, enabled by a cross-manifold optimization technique. 


\section{Related Work}

Joint 3D model/image embeddings: While most shape retrieval methods had traditionally dealt with shape and appearance modalities separately, a recent trend has emerged that exploits the complementary nature of appearance and shape by creating a common representation for images and 3D models. 10. exploits the different advantages of shape and images by using the robustness of 3D models for alignment and pose estimation and the reliability of image labels to identify the objects. While they do not explicitly create a joint manifold based on shape similarity they do rely on image representations for both modalities. Another example of 3D model/image embedding is [15] who first builds a manifold representation of 3D shapes and then trains a CNN to recognize the shape of the object in an image. Unlike our approach, both [1015], limit their representations to objects as a whole preventing the combination of properties taken from different inputs. 23. perform shape retrieval from sketches, words, depth maps and real images by creating a manifold space that combines the different inputs. Since intra-class similarity is not the main focus, most instances of the same class tend to appear clustered. [16] learn a manifold-space metric by using triplets of shapes where the first is similar to the third but dissimilar to the second. Similarly to our approach, the metric space is defined based on shape and not image similarity. 9] first generates voxel representations of the objects present in the RGB image inputs. A shared latent shape representation is then learnt for both images and the voxelized data. At test time RGB convolutions and volume generation deconvolution layers are used to produce the 3D shape. 3D shape blending/mixing: Much in the line of the work presented in this paper, there has been fruitful research in shape blending in recent years. The "3D model evolution" approach of [27] takes a small set of 3D models as input to generate many. Parts from two models cross-over to form a new 3D model, continuing to merge original models with new ones to generate a large number of 3D models. In 1] new shapes are generated by interpolating and varying the topology between two 3D models. The photo-inspired 3D modeling method of 28] takes a single image as input, segments it into parts using an interactive model-driven approach, then retrieves a $3 \mathrm{D}$ model candidate that is finally deformed to match the silhouette of the input photo. The probabilistic approach of [11] learns a model that describes the relationship between the parts of 3D shapes which then allows to create an immense variety of new blended shapes by mixing attributes from different models. The sketch driven method of [26] edits a pre- segmented 3D shape using user-drawn sketches of new parts. The sketch is used to retrieve a matching $3 \mathrm{D}$ part from a catalogue of 3D shapes which is then snapped onto the original 3D shape to create a new blended 3D shape. Note that the above approaches use only 3D shapes as input for shape blending, with the exception of [28] who use a single photograph and [26] who use sketches. However, unlike ours, neither of these approaches can combine different input images to retrieve a shape that blends parts from each input.

Modeling of 3D object parts: We will differentiate between 3D segmentation approaches that seek to ensure consistency in the resulting segmentation across 
different examples of the same object class (co-segmentation) and those that seek a semantically meaningful segmentation (semantic segmentation). Some recent examples of approaches that perform co-segmentation can be found in [24]8], but as we seek to describe parts that have meaning to humans we will focus on the later. We can find examples of semantic 3D parts in approaches like 29]. 229] provides accurate semantic region annotations for large geometric datasets with a fraction of the effort by alternating between using few manual annotations from an expert and a system that propagates labels to new models. We exploit the ShapeNet annotations provided by [29] as the ground truth part shape when constructing our joint manifold.

Recognition of 3D structure from images: The exemplar-based approach of 2 performs joint object category detection viewpoint estimation, exploiting 3D model datasets to render instances from different viewpoints and then learn the combination of viewpoint-instance using exemplar SVMs. 5 uses 3D Convolutional LSTMs to extract the 3D shape of an object from one or more viewpoints. By using LSTM blocks that contain memory, they progressively refine the shape of the object. [7] learn to generate a 3D point cloud from a single RGB image, it learns purely from synthetic data. By using a point cloud instead of a volumetric representation better definition of the details of the shape are obtained. Their novel approach learns how to generate several plausible 3D reconstructions from a single RGB image at test time if the partial observation of the image is ambiguous. 22, learn to recognize the object category and the camera viewpoint for an image using synthetically generated images for training. This work showed that datasets of real images annotated with 3D information were not required to learn shape properties from images as this could be learnt from synthetically generated renderings. 21] obtain good depth estimates for an image given a set of 3D models of the same class.

\section{Overview}

In this section we provide a high level overview of our 3D Pick \& Mix retrieval system. Our system requires a training stage in which: (i) manifolds of 3D shapes of object parts are built (see Fig. (4) and (ii) a CNN is trained to take as input an image and regress the coordinates of each of its constituent parts on the shape manifolds (illustrated in Fig. 22). At query time the system receives an image or set of images as input and obtains the corresponding coordinates on the part manifolds. If the user chooses object parts from different images a cross-manifold optimization is carried out to retrieve a single shape that blends together properties from different images.

Training: At training time, our method takes as input a class-specific collection of 3D shapes (we used ShapeNet [3]) for which part label annotations are available. The first step at training time is to learn a separate shape manifold for each object part (see Fig. 4). Each shape is represented with a Light Field descriptor [4] and characterized with a pyramid of HoG features. The manifolds are then built using non-linear multi-dimensional-scaling (MDS) and the 


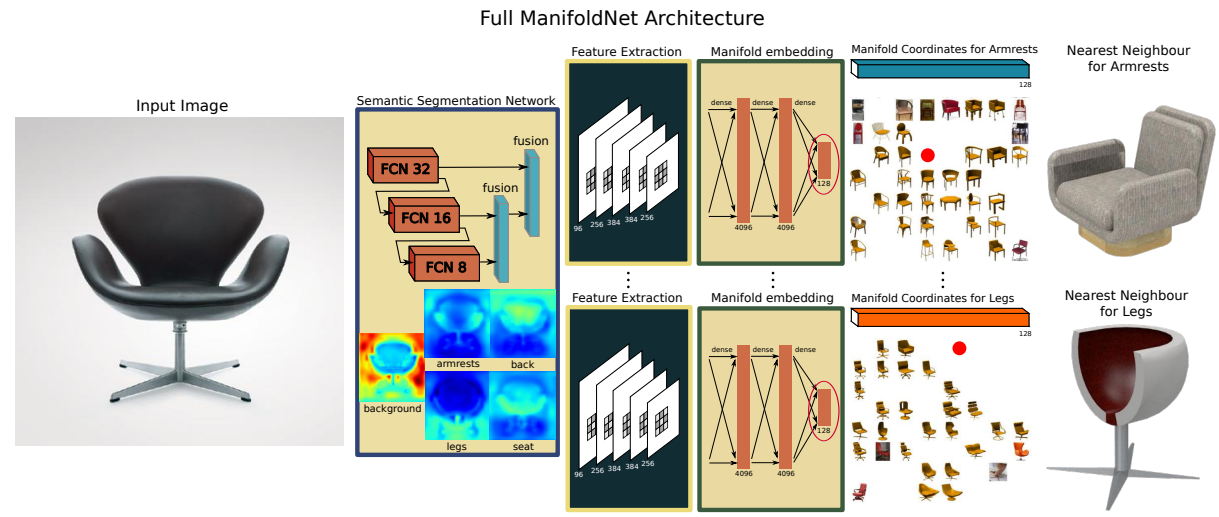

Fig. 2. Summary of the architecture of ManifoldNet, our new deep network that takes an image as input and learns to regress the coordinates of each object part in the different part manifolds. The architecture has 3 sections: the first set of layers performs semantic segmentation of the image pixels into different semantic parts (such as "backrest", "seat", "armrests" or "legs" in the case of chairs). The second section learns an intermediate feature representation for manifold coordinate regression. The final section learns to regress the shape coordinates in each of the part manifolds. We show the nearest neighbour shapes found on the "armrests" and "legs" manifolds for the depicted input image.

$L_{2}$ norm between feature vectors as the distance metric - in each resulting lowdimensional manifold, objects that have similarly shaped parts are close to each other. So far these manifolds of object parts (for instance back-rests, arm-rests, legs, seats in the case of chairs) contain 3D shapes. The second step at training time is to train a CNN to embed images onto each part manifold by regressing their coordinates. We create a set of synthetic training images with per pixel semantic segmentation annotations for the object parts and ground truth manifold coordinates. The architecture of this novel CNN (which we denote ManifoldNet and is shown in Fig. 20 has three clear parts: a set of fully convolutional layers for semantic segmentation of the object into parts; a set of convolutional feature extraction layers; and a set of fully connected layers for manifold coordinate regression. This architecture can be trained end-to-end. We give an example of the produced semantic segmentation in Fig. 3.

Retrieval: At test time, given a new query image of an unseen object, ManifoldNet can embed it into each of the part manifolds by regressing the coordinates. More importantly, our retrieval system can take more than one image as input, picking different object parts from each image. Note that ManifoldNet only needs the input images and the name of the object part that will be used from each image. The network learns jointly to segment the image into parts and to regress the manifold coordinates and therefore it does not require any manual annotations as input. A cross-manifold optimization will then take the coordinates on each of the part manifolds as input and return the coordi- 


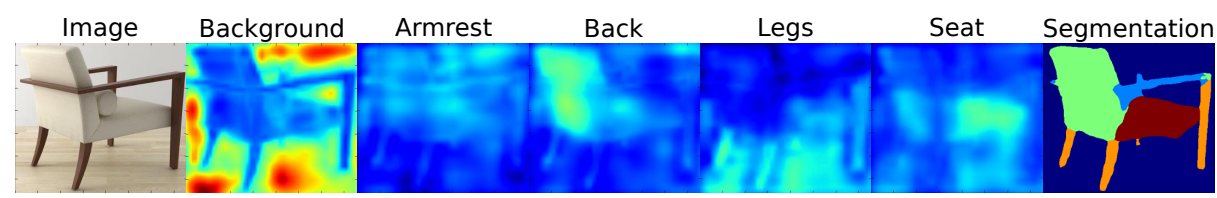

Fig. 3. Example of the semantic segmentation performed by the first stages of our architecture. We can see the output probabilities for each of the parts and the background give a very strong prior of were the parts of an object can be found. Not requiring labels for each part in the input image makes our approach very easy to use and increases dramatically its applicability.

nates of a unique 3D shape that blends the different object parts together. This is achieved through an energy optimization approach, described in section 4.4 .

\section{Methodology}

\subsection{Building shape manifolds for object parts}

We choose to create an embedding space that captures the similarity between the shape of object parts based exclusively on the 3D shapes. The reason behind this choice is that $3 \mathrm{D}$ models capture a more complete, pure and reliable representation of geometry as opposed to images that often display occlusions, or other distracting factors such as texture or shading effects. We then rely on our new CNN architecture to map images onto the same embedding by regressing their coordinates on the corresponding manifolds.

Defining a smooth similarity measure between 3D shapes Shape similarity between object parts is defined as follows. Given a shape $S_{i}$, we define its Light-field Descriptor (LfD) 4] $L_{i}$ as the concatenation of the HoG responses [6] $L_{i}=\left[H_{1} ; H_{2} ; \ldots ; H_{k}\right]$. The value of $k$ is fixed to $k=20$ throughout this work. The light field descriptor $H_{k}$ for each view $k$ is defined as $H_{k}=\left[H_{k}^{\text {mid }} ; H_{k}^{\text {low }}\right] \in \mathbb{R}^{2610}$. The $L_{2}$ distance between feature vectors is then used as the similarity measure between a pair of shapes $S_{i}$ and $S_{j}: d_{i j}=\left\|L_{i}-L_{j}\right\|_{2}$ where $L_{i} \in \mathbb{R}^{52200}$. We found that using only the mid and low frequency parts of the HoG pyramid leads to smoother transitions in shape similarity. For this reason we do not use the original 3 level HoG pyramids but just the 2 higher levels of the pyramids. This allows for smooth transitions in shape similarity between parts making the shape blending possible. Due to most 3D models available in Internet datasets not being watertight but only polygon soups we are required to use projective shape features like [4. We now build separate manifolds for each object part. Each shape $S_{i}$ is therefore split into its constituent parts $\forall S_{i}: \exists\left\{S_{i}^{1} ; S_{i}^{2} ; \ldots ; S_{i}^{P}\right\}$, where $P$ is the total number of parts and $S_{i}^{p}$ is the shape of part $p$ of object $i$. If a part is not present in an object (for instance, chairs without arm-rests) we set all the components of the vector $L_{i}^{p}$ to zero, which is equivalent to computing the HoG descriptor of an empty image. 


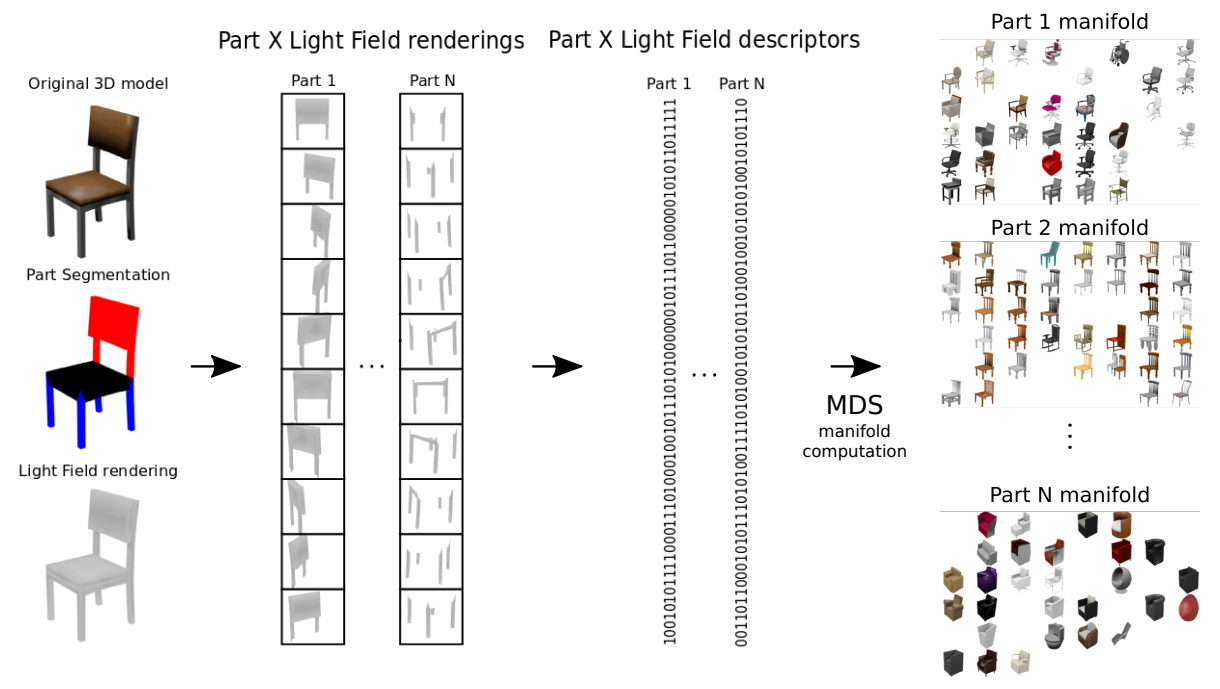

Fig. 4. Shape manifold construction. The shape of each part for each $3 \mathrm{D}$ model is rendered from different viewpoints and represented with a Light Field descriptor 44. The manifolds are then built using non-linear multi-dimensional-scaling (MDS) and the $L_{2}$ norm between feature vectors as the distance metric. In each low-dimensional manifold, objects that have similarly shaped parts appear close to each other.

\subsection{Building shape manifolds of parts}

Using the similarity measure between the shape of object parts we use it to construct a low dimensional representation of the shape space. In principle, the original $L_{i} \in \mathbb{R}^{52200}$ feature vectors could have been used to represent each shape, since distances in that space reflect well the similarity between shapes. We reduce the dimensionality from 52,200 to 128 dimensions and we use nonlinear Multi-Dimensional Scaling (MDS) [14 to build the shape manifolds. We compute the distance matrix $D^{p} \in \mathbb{R}^{n \times n}$ as $D^{p}(i, j)=d_{i j}^{p}$, were $p$ is the index of the part and $n$ is the total number of shapes. The manifold is built using MDS by minimizing a Sammon Mapping error [19] defined as

$$
E^{p}=\frac{1}{\sum_{i<j} D^{p}(i, j)} \sum_{i<j} \frac{\left(D^{p}(i, j)-D^{\prime p}(i, j)\right)^{2}}{D^{p}(i, j)}
$$

where $D^{p}$ is the distance matrix between shapes in the original high dimensional feature space $L^{p}$; and $D^{\prime p}$ is the distance matrix between shapes in the new low dimensional manifold $L^{\prime p}$. With the different manifolds $L^{\prime p}$ for each part $p$ computed, a low dimensional representation of shape similarity exists and all 3D shapes are already included in it. Adding new 3D shapes to the manifold is done by solving an optimization that minimizes the difference between the distances between all previous shapes and the new shape in $D^{p}$ and the distances in $D^{\prime p}$ with respect to the predicted embedding point. To understand the shape of 


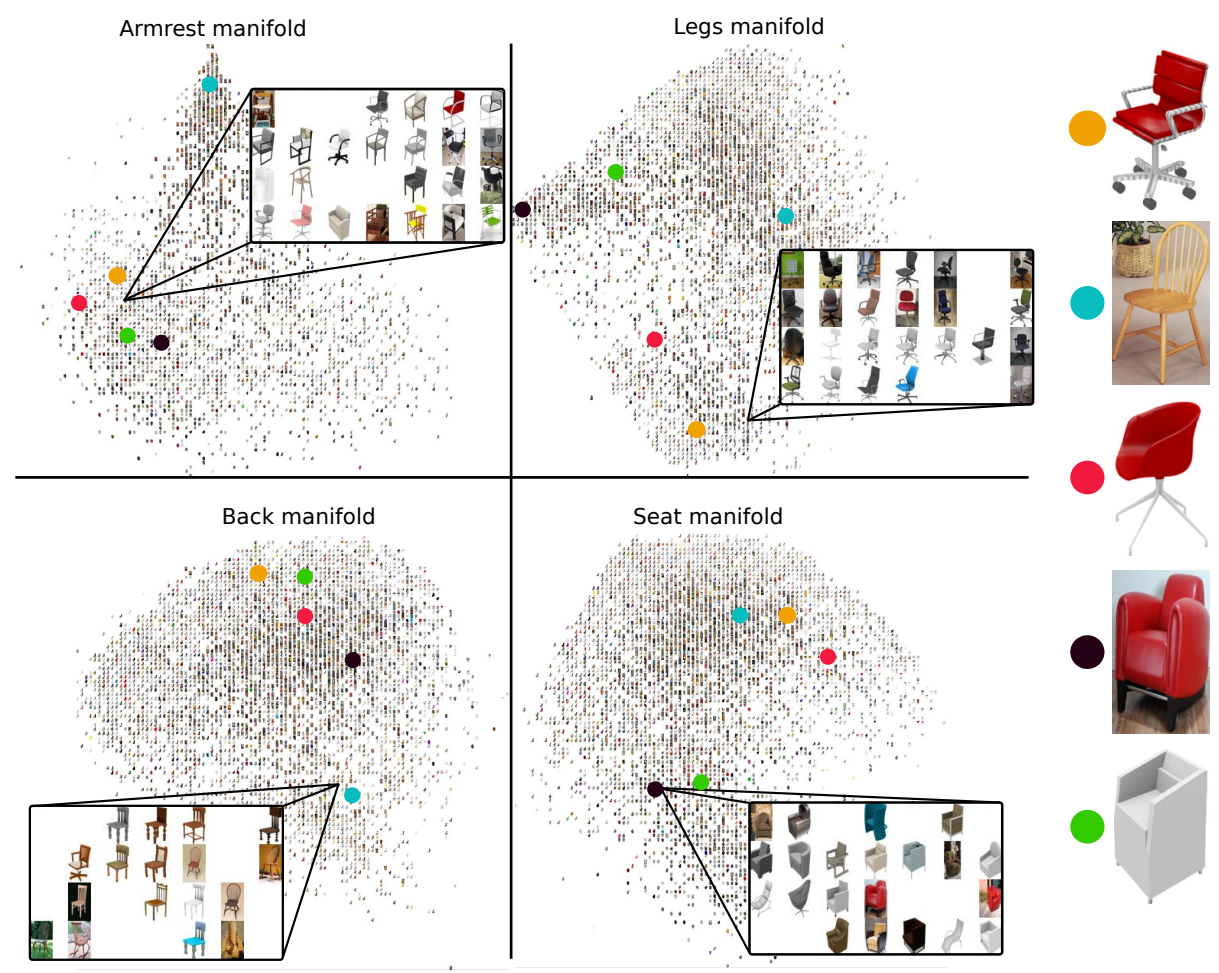

Fig. 5. Two dimensional visualizations of the four low dimensional part manifolds "backrest", "seat", "armrests" and "legs" for the chairs class. Probabilistic PCA has been used to provide a $2 \mathrm{D}$ visualization of the 128 dimensional manifolds. Both images and 3D models have been represented in the manifolds. Objects with similarly shaped parts lie close to each other on the manifold. Several shapes are tagged in all four manifolds to show how vicinity changes for each part. All shapes and images exist in all manifolds.

the produced manifolds we provide a 2D visualization in Fig. 5 . In this figure we can better understand how the manifolds relate the different parts of an object.

\subsection{Learning to embed images into the shape manifolds}

Building the shape manifolds $L^{\prime p}$ for each part $p$ based only on 3D models, we have successfully abstracted away effects such as textures, colours or materials. The next step is to train a deep neural network that can map RGB images onto each manifold by regressing the coordinates on each part manifold directly from RGB inputs. Crucially, the input to the network must be simply the RGB image and the name (label) of the object part $p$ selected from that image - for instance embed this image of a chair into the manifold of "chair legs".

We propose a novel deep learning architecture, which we call ManifoldNet, it performs three tasks: first, it learns how to estimate the location of different 


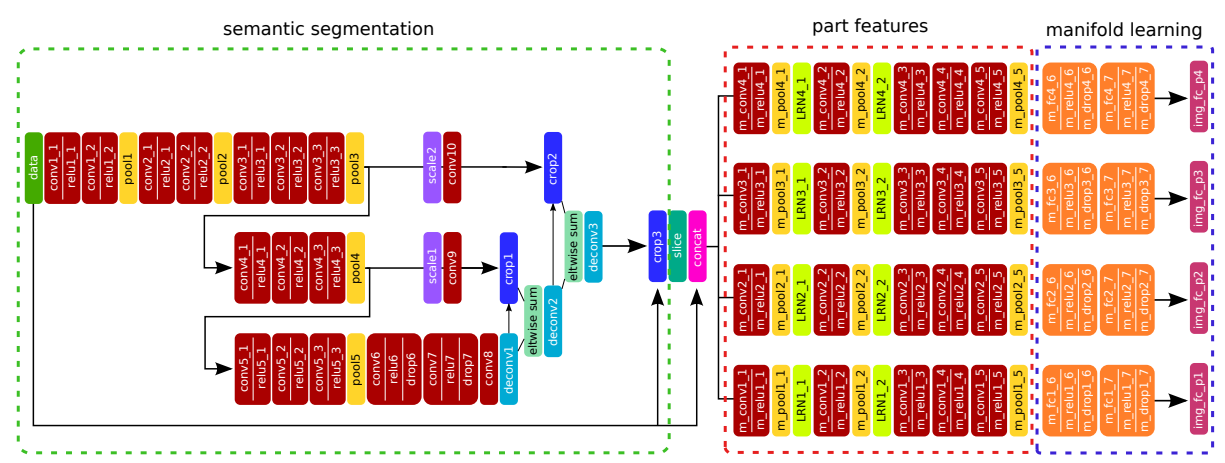

Fig. 6. Detailed ManifoldNet architecture. The first set of layers take care of assigning semantic part labels to each of the pixels in the image. The second stage extracts intermediate features to help the manifold learning. The final stage uses a set of fully convolutional layers to regress the manifold coordinates.

parts in the image by performing semantic segmentation, it then uses the semantic labeling and the original input image to learn $p$ different intermediate feature spaces for each object part and finally, $p$ different branches of fully connected layers will learn the final image embedding into the respective part shape manifold. The network has a general core that performs semantic segmentation and specialized branches for each of the manifolds in a similar fashion as [13.

ManifoldNet: A multi-manifold learning architecture A summary of our new architecture is shown in Fig. 2 and a detailed description of all the layers in Fig. 6. The common part of the architecture, which performs the semantic segmentation, is a fully convolutional approach closely related to [17. The fully convolutional architecture uses a VGG-16 architecture [20 for its feature layers. A combination of FCN-32, FCN-16 and FCN-8 is used to obtain more detailed segmentations but all sub-parts are trained together for simplicity.

The other two parts of the architecture shown in Fig. 2 take care of: (1) creating an intermediate feature space, and (2) learning the manifold embedding. The intermediate feature layers take as input the concatenation of the original RGB image and the heat maps given as output by the semantic segmentation layers to learn a feature representation that eases the manifold learning task. Finally, the manifold coordinate regression module is formed by 3 fully connected layers (the first two use relu non-linearities). A dropout scheme is used to avoid over-fitting to the data. Trained models and code are already public but due to anonymity we cannot disclose the URL.

Details of the training strategy The training of such a deep architecture requires careful attention. First, to avoid vanishing gradients and to improve convergence, the semantic segmentation layers are trained independently by using a standard cross-entropy classification loss: 


$$
L_{\text {seg }}=\frac{-1}{N} \sum_{n=1}^{N} \log \left(p_{n, l_{n}}\right)
$$

where $p_{n, l_{n}}$ is the softmax output class probability for each pixel. A batch size of only 20 is used at this stage due to memory limitations on the GPU and the high number of weights to be trained.

When trying to train the manifold layers we found out that convergence heavily depended on big batch sizes and many iterations. At this point we used a learning scheme that allowed us to have bigger batch sizes during training and faster computation of each iteration. The trick is quite simple really, we precompute for all training images the output of the semantic layers and only train the part branches of the network. By doing this we are training a substantially shallower network allowing for significantly bigger batch sizes. The network is trained by minimizing the following euclidean loss:

$$
L_{\text {mani }}=\frac{1}{2 N} \sum_{i=1}^{N}\left\|x_{e s t}^{i}-x_{g t}^{i}\right\|_{2}^{2}
$$

where $x_{e s t}^{i}$ are the manifold coordinates estimated by the network and $x_{g t}^{i}$ are the ground truth manifold coordinates. The Euclidean loss is chosen since the part shape manifolds are themselves Euclidean spaces. With this good initialization of the weights we finally perform an end-to-end training of all layers using only the final euclidean loss.

Training data and data augmentation: the training images are generated synthetically by rendering models from ShapeNet [3]. We use the 3D part annotations on the 3D models, available from [29, to provide ground truth values for the semantic segmentation. We generate 125 training images per model from different poses, and a random RGB image taken from the Pascal 3D 25] dataset is added as background. To recap, the proposed approach is invariant to pose and manages to learn solely from rendered synthetic images.

\subsection{Shape blending through cross-manifold optimization}

Once the manifold coordinates for the different object parts have been estimated all the information needed to blend them into a single 3D model is available. We formulate this as a 3D shape retrieval problem: "find the 3D model, from the existing shapes represented in the manifolds, that best fits the arrangement of parts". The user selects two (or more) images (or 3D models) and indicates the part they wish to select from each one (note that no annotations are needed, only the name/label of the part). The cross-manifold optimization now finds the 3D shape in the collection that minimizes the sum of the distances to each of the parts. In more detail - first, all manifolds need to be normalized to allow a meaningful comparison of distances. Then, given the set of manifold coordinates for the selected parts, a shape prediction $b$ can be defined as the concatenation of the respective part coordinates $b=\left\{b^{1} ; \ldots ; b^{m}\right\}$. The goal is now to retrieve 
a 3D model from the shape collection whose coordinates $a=\left\{a^{1} ; \ldots ; a^{m}\right\}$ are closest to this part arrangement by minimizing the following distance:

$$
B=\min _{a \in \mathbb{S}} \sum_{k=1}^{m}\left\|a^{m}-b^{m}\right\|
$$

where $\mathbb{S}$ is the set of existing shapes. Note that not all parts need to be selected to obtain a blended shape, we define $m$ as the subset of parts to be blended, where $m \subseteq p$. Also, notice that blending can be done by combining any number of parts from any number of sources (shapes/images).

\section{Results}

We perform a set of qualitative and quantitative experiments to evaluate the performance of our approach. Allthough our approach performs shape blending from several inputs this can be understood as a retrieval task.

\subsection{Quantitative results on Image-based Shape Retrieval}

The proposed approach will be at a disadvantage when trying to retrieve whole shapes and the same will happen when approaches that model the object as a whole try to retrieve parts. What has been have done is a experimental comparison of both approaches on both tasks, as a clean unbiased comparison cannot be done on a single experiment both approaches will be used to solve both tasks. This is possible as all compared approaches can be used as a similarity measure between images and 3D models. By doing this, how much is lost can be measured when either modeling the whole object or the individual parts.

Image-based Whole Shape Retrieval We perform whole shape image-based retrieval on the ExactMatch dataset [15. The experiment compares against Li [15, a state-of-the-art deep volumetric approach in Girdhar [9], HoG, AlexNet and Siamese networks. Two versions of the proposed approach using the original three level HoG pyramid features to build the manifold and the two level HoG manifold features that have been shown to be better fitted for a smoother shape similarity measure. Our approach predicts the part coordinates separately in each of the part manifolds. The estimations of each part are then used to solve the blending optimization and obtain a single shape prediction. The fact that the neural network estimates the coordinates individually means that all the part co-occurrence information that is implicitly encoded in the approaches that model the object as a whole is lost during training, nevertheless, the proposed approach can still yield good results that are comparable to those that model the whole shape. It can also be seen that for the exact shape retrieval the original three level pyramid HoG features perform better which is to be expected. The results of these experiments are included in Fig. 7 (first image). 

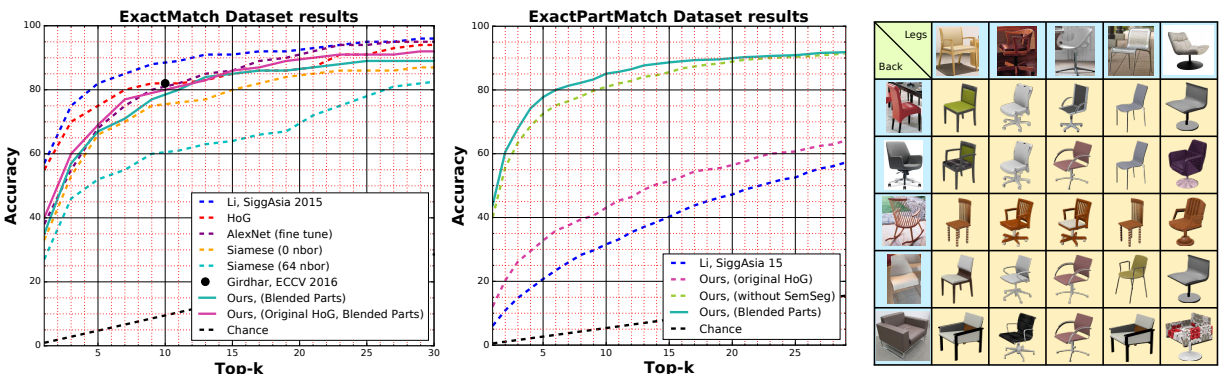

Fig. 7. Left: Image-based whole shape retrieval results obtained on the ExactMatch dataset. Center: Shape blending and retrieval from image-based part descriptions on the ExactPartMatch dataset. Right: Matrix showing combination results. Experiment performed by generating all possible combinations of legs and backs from 10 test shapes.

Image-based Part Shape Blending To test the performance of part blending and retrieval a new dataset has been created using a subset of the shapes from the Shapenet database [3] and the images from the ExactMatch dataset [15] to create the ExactPartMatch dataset. The task is to find the correct 3D shape out of all the annotated 3D models using the parts from the specified inputs. As in Shapenet many of the 3D models are repeated many times (e.g. ikea chairs) we need to control that there is only one correct match with the part mix. The dataset contains $\mathbf{8 4 5}$ test cases. Each test case if assigned to one of 187 hand annotated ground truth 3D models, the same part shape combination is tested using images from different angles, textures and lighting conditions. Each test case is a combination of parts from two different images for which a $3 D$ model exists in Shapenet. There are examples of all possible part combinations. The whole dataset is already public online, the link is not disclosed due to anonymity. The results of these experiments are included in Fig. 7 (second image). Also in Fig. 7 (third image) we include an experiment that shows the product of crossing 5 leg samples with 5 back samples densely, this shows the kind of results to expect from our approach.

For all approaches similarity is estimated in shape manifold space and then the multi-manifold optimization is performed, which is required to obtain the most similar shape. The approaches shown are Ours, with and without semantic segmentation, Ours with semantic segmentation but using the original three level HoG features, Li'sSiggAsia15 [15] and random chance. [15] struggles to get results as good as the ones obtained by modeling the parts. Their holistic representation enables them to better model a whole object but loose substantial performance when trying to identify individually the parts. In contrast our approach looses the information of part co-occurrence in favour of being capable to model the parts individually. Also, exploiting the semantic segmentation of the input is consistently better as it defines the actual interest zones in the image. If considering top- 5 results without semantic segmentation $65 \%$ is obtained but when using semantic segmentation $76 \%$ is obtained, which is a substantial $11 \%$ improvement in performance. It can also be seen that trying to blend shapes 


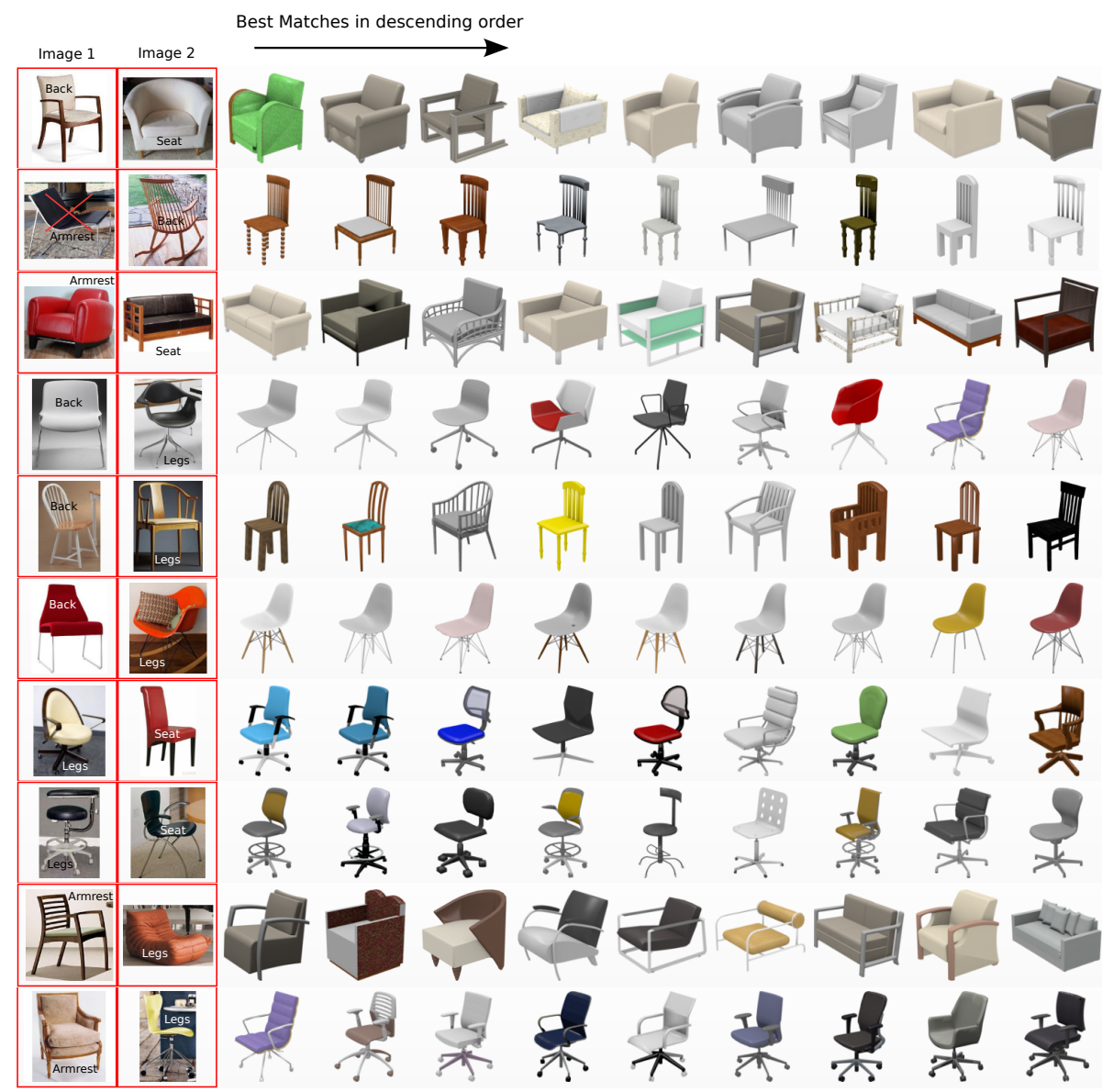

Fig. 8. Qualitative results extracted to show performance on different part arrangements using parts from two different image inputs. The results show the closest matching shapes in the Shapenet dataset. If the part is not present, like in the second row, an $X$ in the colour of the desired part is used to label the non-presence of that part.

when using shape similarities that do not correctly model smoothness over shape has a tremendous impact in performance (Ours_original_HoG).

\subsection{Qualitative results on Image-based Shape Retrieval}

To further asses the quality of the results examples are shown depicting the performance of the approach being applied to real images. In Fig. 8 and in Fig. 9 many example images taken from the ExactPartMatch dataset detailed in previous sections are shown. Our approach searches over the entirety of Shapenet to find the closest matches to the input part arrangement. Many different part arrangements are accounted for in the figure to show that the proposed approach 


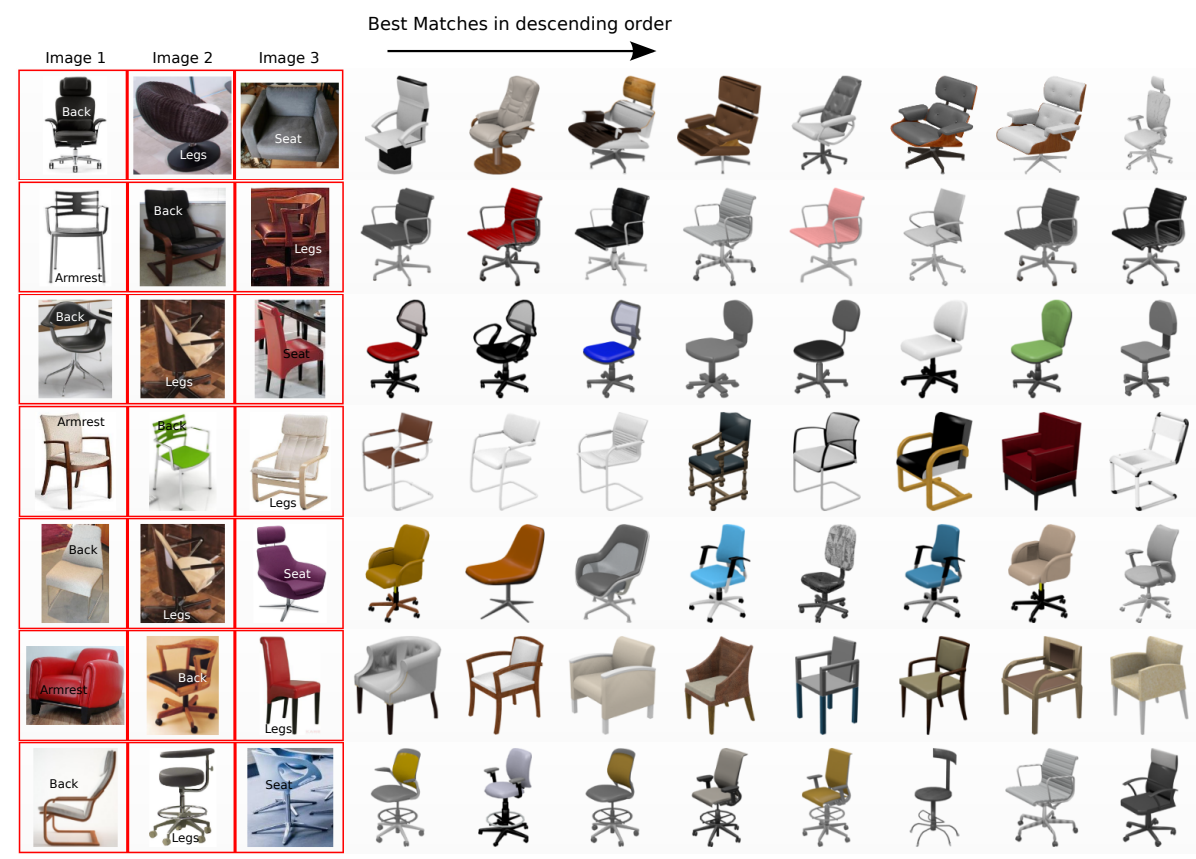

Fig. 9. Qualitative results extracted to show performance on different part arrangements using parts from three different image inputs. The results show the closest matching shapes in the Shapenet dataset.

can capture not only the big differences but also more subtle differences like the number of legs in the base of a specific swivel chair, the fact that a wooden chair with a back made of bars has a round top or a flat top, capturing the detailed shape of the interconnecting supports of the four legs, etc.

\section{Conclusions}

An approach capable of modelling and blending object parts from images and 3D models has been presented. This approach has demonstrated that by using a common manifold representation very elaborate queries can be done in massive Internet databases. It has also shown to be capable to produce accurate shape retrieval which proves its understanding of the underlying shapes. This provides a natural link between shape and image datasets and opens numerous possibilities of application to similar tasks. Also, by understanding the parts that give semantic meaning to an object volumetric approaches like [5]12]18/7] could potentially address their difficulties to produce details in their volumetric estimations by either enforcing the shape of the part explicitly or balancing the voxel occupancy probabilities for each part independently. 


\section{References}

1. Alhashim, I., Li, H., Xu, K., Cao, J., Ma, R., Zhang, H.: Topology-varying 3d shape creation via structural blending. ACM Transactions on Graphics (2014)

2. Aubry, M., Maturana, D., Efros, A., Russell, B., Sivic, J.: Seeing 3d chairs: exemplar part-based 2d-3d alignment using a large dataset of cad models. Computer Vision and Pattern Recognition (CVPR) (2014)

3. Chang, A.X., Funkhouser, T., Guibas, L., Hanrahan, P., Huang, Q., Li, Z., Savarese, S., Savva, M., Song, S., Su, H., Xiao, J., Yi, L., Yu, F.: ShapeNet: An Information-Rich 3D Model Repository. Tech. Rep. arXiv:1512.03012 [cs.GR], Stanford University - Princeton University — Toyota Technological Institute at Chicago (2015)

4. Chen, D.Y., Tian, X.P., Shen, Y.T., Ouhyoung, M.: On visual similarity based 3d model retrieval. Computer Graphics Forum (2003)

5. Choy, C.B., Xu, D., Gwak, J., Chen, K., Savarese, S.: 3d-r2n2: A unified approach for single and multi-view $3 d$ object reconstruction. European Conference on Computer Vision (ECCV) (2016)

6. Dalal, N., Triggs, B.: Histograms of oriented gradients for human detection. In: Computer Vision and Pattern Recognition (CVPR) (2005)

7. Fan, H., Su, H., Guibas, L.J.: A point set generation network for 3d object reconstruction from a single image. Computer Vision and Pattern Recognition (CVPR) (2017)

8. Fish, N., van Kaick, O., Bermano, A., Cohen-Or, D.: Structure-oriented networks of shape collections. ACM Transactions on Graphics (2016)

9. Girdhar, R., Fouhey, D., Rodriguez, M., Gupta, A.: Learning a predictable and generative vector representation for objects. European Conference on Computer Vision (ECCV) (2016)

10. Hueting, M., Ovsjanikov, M., Mitra, N.: Crosslink: Joint understanding of image and $3 \mathrm{~d}$ model collections through shape and camera pose variations. ACM Transactions on Graphics (Proc. SIGGRAPH Asia) (2015)

11. Kalogerakis, E., Chaudhuri, S., Koller, D., Koltun, V.: A probabilistic model for component-based shape synthesis. ACM Transactions on Graphics (2012)

12. Kar, A., Tulsiani, S., Carreira, J., Malik, J.: Category-specific object reconstruction from a single image. Computer Vision and Pattern Recognition (CVPR) (2015)

13. Kokkinos, I.: Ubernet: Training a 'universal' convolutional neural network for low-, mid-, and high-level vision using diverse datasets and limited memory. In: Computer Vision and Pattern Recognition (CVPR) (2017)

14. Kruskal, J.B.: Multidimensional scaling by optimizing goodness of fit to a nonmetric hypothesis. Psychometrika (1964)

15. Li, Y., Su, H., Qi, C.R., Fish, N., Cohen-Or, D., Guibas, L.J.: Joint embeddings of shapes and images via cnn image purification. ACM Trans. Graph. (2015)

16. Lim, I., Gehre, A., Kobbelt, L.: Identifying style of 3d shapes using deep metric learning. Computer Graphics Forum (2016)

17. Long, J., Shelhamer, E., Darrell, T.: Fully convolutional networks for semantic segmentation. In: Computer Vision and Pattern Recognition (CVPR) (2015)

18. Qi, C.R., Su, H., Niessner, M., Dai, A., Yan, M., Guibas, L.J.: Volumetric and multi-view cnns for object classification on 3d data. Computer Vision and Pattern Recognition (CVPR) (2016)

19. Sammon, J.W.: A nonlinear mapping for data structure analysis. IEEE Transactions on Computers (1969) 
20. Simonyan, K., Zisserman, A.: Very deep convolutional networks for large-scale image recognition. International Conference on Learning Representations (2015)

21. Su, H., Huang, Q., Mitra, N.J., Li, Y., Guibas, L.: Estimating image depth using shape collections. ACM Transactions on Graphics (2014)

22. Su, H., Qi, C.R., Li, Y., Guibas, L.J.: Render for cnn: Viewpoint estimation in images using cnns trained with rendered $3 \mathrm{~d}$ model views. In: International Conference on Computer Vision (ICCV) (2015)

23. Tasse, F.P., Dodgson, N.: Shape2vec: Semantic-based descriptors for 3d shapes, sketches and images. ACM Transactions on Graphics (2016)

24. Tulsiani, S., Su, H., Guibas, L.J., Efros, A.A., Malik, J.: Learning shape abstractions by assembling volumetric primitives. Computer Vision and Pattern Recognition (CVPR) (2017)

25. Xiang, Y., Mottaghi, R., Savarese, S.: Beyond pascal: A benchmark for 3d object detection in the wild. In: IEEE Winter Conference on Applications of Computer Vision (WACV) (2014)

26. Xie, X., Xu, K., Mitra, N.J., Cohen-Or, D., Gong, W., Su, Q., Chen, B.: Sketchto-Design: Context-Based Part Assembly. Computer Graphics Forum (2013)

27. Xu, K., Zhang, H., Cohen-Or, D., Chen, B.: Fit and diverse: Set evolution for inspiring 3d shape galleries. ACM Transactions on Graphics (2012)

28. Xu, K., Zheng, H., Zhang, H., Cohen-Or, D., Liu, L., Xiong, Y.: Photo-inspired model-driven 3d object modeling. ACM Transactions on Graphics (2011)

29. Yi, L., Kim, V.G., Ceylan, D., Shen, I.C., Yan, M., Su, H., Lu, C., Huang, Q., Sheffer, A., Guibas, L.: A scalable active framework for region annotation in 3d shape collections. SIGGRAPH Asia (2016) 